\title{
Baseline Clinical and Biomarker Characteristics of Biobank Innovations for Chronic Cerebrovascular Disease With Alzheimer's Disease Study: BICWALZS
}

\author{
Hyun Woong Roh ${ }^{1}$, Na-Rae Kim ${ }^{1}$, Dong-gi Lee ${ }^{1}$, Jae-Youn Cheong ${ }^{2,3}$, Sang Won Seo ${ }^{4}$, Seong Hye Choi, \\ Eun-Joo Kim ${ }^{6}$, Soo Hyun $\mathrm{Cho}^{7}$, Byeong C. Kim ${ }^{7}$, Seong Yoon $\mathrm{Kim}^{8}$, Eun Young Kim ${ }^{9}$, Jaerak Chang ${ }^{9}$, \\ Sang Yoon Lee ${ }^{10}$, Dukyong Yoon ${ }^{11}$, Jin Wook Choi ${ }^{12}$, Young-Sil An ${ }^{13}$, Hee Young Kang ${ }^{14}$, \\ Hyunjung Shin ${ }^{15,16}$, Bumhee Park ${ }^{17,18}$, Sang Joon Son ${ }^{1}$, and Chang Hyung Hong ${ }^{1}$ \\ ${ }^{1}$ Department of Psychiatry, Ajou University School of Medicine, Suwon, Republic of Korea \\ ${ }^{2}$ Department of Gastroenterology, Ajou University School of Medicine, Suwon, Republic of Korea \\ ${ }^{3}$ Human Genome Research and Bio-Resource Center, Ajou University Medical Center, Suwon, Republic of Korea \\ ${ }^{4}$ Department of Neurology, Samsung Medical Center, Sungkyunkwan University School of Medicine, Seoul, Republic of Korea \\ ${ }^{5}$ Department of Neurology, Inha University School of Medicine, Incheon, Republic of Korea \\ ${ }^{6}$ Department of Neurology, Pusan National University Hospital, Pusan National University School of Medicine and Medical Research Institute, \\ Busan, Republic of Korea \\ ${ }^{7}$ Department of Neurology, Chonnam National University Medical School, Chonnam National University Hospital, Gwangju, Republic of Korea \\ ${ }^{8}$ Department of Psychiatry, Asan Medical Center, University of Ulsan College of Medicine, Seoul, Republic of Korea \\ ${ }^{9}$ Department of Brain Science, Ajou University School of Medicine, Suwon, Republic of Korea \\ ${ }^{10}$ Department of Biomedical Sciences, Ajou University Graduate School of Medicine, Suwon, Republic of Korea \\ ${ }^{11}$ Department of Biomedical Systems Informatics, Yonsei University College of Medicine, Yongin, Republic of Korea \\ ${ }^{12}$ Department of Radiology, Ajou University School of Medicine, Seoul, Republic of Korea \\ ${ }^{13}$ Department of Nuclear Medicine and Molecular Imaging, Ajou University School of Medicine, Suwon, Republic of Korea \\ ${ }^{14}$ Department of Dermatology, Ajou University School of Medicine, Suwon, Republic of Korea \\ ${ }^{15}$ Department of Industrial Engineering, Ajou University, Suwon, Republic of Korea \\ ${ }^{16}$ Department of Artificial Intelligence, Ajou University, Suwon, Republic of Korea \\ ${ }^{17}$ Department of Biomedical Informatics, Ajou University School of Medicine, Suwon, Republic of Korea \\ ${ }^{18}$ Office of Biostatistics, Ajou Research Institute for Innovative Medicine, Ajou University Medical Center, Suwon, Republic of Korea
}

Objective We aimed to present the study design and baseline cross-sectional participant characteristics of biobank innovations for chronic cerebrovascular disease with Alzheimer's disease study (BICWALZS) participants.

Methods A total of 1,013 participants were enrolled in BICWALZS from October 2016 to December 2020. All participants underwent clinical assessments, basic blood tests, and standardized neuropsychological tests $(n=1,013)$. We performed brain magnetic resonance imaging (MRI, $\mathrm{n}=817$ ), brain amyloid positron emission tomography (PET, $\mathrm{n}=713$ ), single nucleotide polymorphism microarray chip (K-Chip, $n=949$ ), locomotor activity assessment (actigraphy, $n=200$ ), and patient-derived dermal fibroblast sampling $(n=175)$ on a subset of participants.

Results The mean age was 72.8 years, and 658 (65.0\%) were females. Based on clinical assessments, total of 168, 534, 211, 80, and 20 had subjective cognitive decline, mild cognitive impairment (MCI), Alzheimer's dementia, vascular dementia, and other types of dementia or not otherwise specified, respectively. Based on neuroimaging biomarkers and cognition, 199, 159, 78, and 204 were cognitively normal $(\mathrm{CN})$, Alzheimer's disease $(\mathrm{AD})$-related cognitive impairment, vascular cognitive impairment, and not otherwise specified due to mixed pathology (NOS). Each group exhibited many differences in various clinical, neuropsychological, and neuroimaging results at baseline. Baseline characteristics of BICWALZS participants in the MCI, AD, and vascular dementia groups were generally acceptable and consistent with 26 worldwide dementia cohorts and another independent AD cohort in Korea.

Conclusion The BICWALZS is a prospective and longitudinal study assessing various clinical and biomarker characteristics in older adults with cognitive complaints. Details of the recruitment process, methodology, and baseline assessment results are described in this paper.

Psychiatry Investig 2022;19(2):100-109

Keywords Alzheimer's disease; Vascular dementia; Subcortical vascular cognitive impairment; Mild cognitive impairment; Dementia; Cohort.

\footnotetext{
Received: October 19, 2021 Accepted: November 3, 2021

$\triangle$ Correspondence: Sang Joon Son, MD, PhD

Department of Psychiatry, Ajou University School of Medicine, 206 World cup-ro, Yeongtong-gu, Suwon 16499, Republic of Korea

Tel: +82-31-219-7850, Fax: +82-31-219-5179, E-mail: sjsonpsy@ajou.ac.kr

Correspondence: Chang Hyung Hong, MD, PhD

Department of Psychiatry, Ajou University School of Medicine, 206 World cup-ro, Yeongtong-gu, Suwon 16499, Republic of Korea
}

Tel: +82-31-219-5180, Fax: +82-31-219-5179, E-mail: antiaging@ajou.ac.kr

(c) This is an Open Access article distributed under the terms of the Creative Commons Attribution Non-Commercial License (https://creativecommons.org/licenses/by-nc/4.0) which permits unrestricted non-commercial use, distribution, and reproduction in any medium, provided the original work is properly cited 


\section{INTRODUCTION}

Alzheimer's disease $(\mathrm{AD})$ is the most common cause of dementia. Nevertheless, the underlying mechanisms and pathophysiology of $\mathrm{AD}$ are not fully understood, and there is currently no effective treatment for AD. ${ }^{1}$ Regarding the disappointing outcome of the disease-modifying trials of $\mathrm{AD},{ }^{2-4}$ researchers have pointed out that mixed pathology of amyloid pathology, vascular pathology, tau pathology, and/or alpha-synuclein pathology may be a possible cause.,

Recent studies have shown that cortical amyloid burden, a hallmark of $\mathrm{AD}$, frequently occurs alongside chronic cerebrovascular disease (CVD), such as white matter hyperintensities (WMH), lacunar infarction, and/or microbleeds in patients with cognitive decline. ${ }^{7,8}$ Recent studies suggest that progressive cognitive decline can be driven by amyloid burden or vascular burden alone but can also be driven by the presence of both pathologies and their synergistic effects on neurodegeneration. ${ }^{9-12}$ In this context, the cortical amyloid burden and vascular burden caused by chronic CVD have become important research goals over the last decade. Given the above, the Biobank Innovations for Chronic Cerebrovascular Disease with ALZheimer's Disease Study (BICWALZS) was planned and initiated in 2016 by the Korea Disease Control and Prevention Agency for the Korea Biobank Project. The BICWAL$\mathrm{ZS}$ is a multi-center collaborative research project with five clinical centers, a regional biobank, and the National Biobank of Korea.

The original goal of BICWALZS was to facilitate, regulate, and ensure optimal research use of human biospecimens and data in the field of $\mathrm{AD}$, chronic CVD, and mixed pathology for mild cognitive impairment (MCI) and dementia. Considering the multifactorial nature of neurodegenerative disorders and their heterogeneous clinical presentation, it is essential to assess not only clinical characteristics but also various biomarkers such as neuroimaging results, genomics data using single nucleotide polymorphism microarray chips, and actigraphy measurements. ${ }^{13}$ In addition, considering its potential for the generation of induced pluripotent stem cells, possible ex vivo research, and precision medicine, we also sampled patient-derived dermal fibroblast in a subset of participants. ${ }^{14,15}$

This paper reports the study design, methodology, and baseline clinical and biomarker characteristics of BICWALZS.

\section{METHODS}

\section{Participants}

BICWALZS was planned and initiated in October 2016 by the Korea Disease Control and Prevention Agency for the Korea Biobank Project which is a national innovative bio- banking program to foster biomedical and healthcare R\&D infrastructure. A total of five institutions participated: Ajou University Hospital, Samsung Medical Center, Inha University Hospital, Pusan National University Hospital, and Chonnam National University Hospital. The multi-site infrastructure is linked to a centralized coordinator center and database. A coordinating committee is in place to harmonize and standardize specimen handling, technical best practices, data entry, management, and ethical and legal issues. A total of 1013 participants were enrolled in the BICWALS from October 2016 to December 2020. All participants underwent clinical assessments, blood tests, and standardized neuropsychological tests $(n=1,013)$. From the subset of the participants, we performed brain magnetic resonance imaging (MRI, $n=817$ ), brain amyloid positron emission tomography (PET, $n=713$ ), single nucleotide polymorphism microarray chip ( $\mathrm{n}=949)$, actigraphy measurement $(n=200)$, and patient-derived dermal fibroblast sampling ( $\mathrm{n}=175)$. We planned to follow all participants annually with brief assessments and participants who had cortical amyloid burden, subcortical vascular pathology, apolipoprotein E4 allele, or significant cognitive decline biannually with an expanded assessment including neuropsychological tests, actigraphy measurements, and brain MRI. We first classified the participants according to their clinical diagnosis. The subjective cognitive decline (SCD) criteria included self and/or informant reports of cognitive decline but no impairment in objective cognitive tests and daily functioning. Participants with MCI were evaluated using the expanded Mayo Clinic criteria. ${ }^{16}$ Participants with Alzheimer's dementia met the core clinical criteria suggested by the National Institute on Aging and the Alzheimer's Association working group in $2011 .{ }^{17}$ Participants with vascular dementia were evaluated using major vascular neurocognitive disorder criteria, as suggested in the fifth edition of the Diagnostic and Statistical Manual of Mental Disorders. ${ }^{18}$ Additionally, for the neuroimaging-based approach and our study purpose, we classified a subset of participants into $\mathrm{CN}, \mathrm{ADCI}$, or VCI based on the mini-mental state examination (MMSE) and two critical neuroimaging biomarkers, cortical amyloid burden and subcortical vascular burden. For this process, age, sex, and education matched -1, or lower $\mathrm{z}$-scores were considered cognitive decline. As a result, participants with amnestic MCI or AD were considered to have ADCI. Participants with possible other vascular pathologies, such as severe white matter intensities, were excluded from the ADCI group. Participants with subcortical vascular MCI or subcortical vascular dementia were considered to have an VCI. Participants with cortical amyloid pathology on amyloid PET or those with infarctions or WMH due to radiation injury, multiple sclerosis, vasculitis, or leukodystrophy were excluded from $\mathrm{CN}$ and VCI group. 
BICWALZS is registered in the Korean National Clinical Trial Registry CRIS (identifier: KCT0003391). Prior to beginning the study, the Institutional Review Board approved the study plan (AJIRB-BMR-SUR-16-362). Written informed consent was obtained from all participants and caregivers. The study was conducted in accordance with the International Harmonization Conference guidelines on Good Clinical Practice.

\section{Neuropsychological test}

Cognitive function was evaluated using a standardized neuropsychological test, Seoul neuropsychological screening battery (SNSB). ${ }^{19}$ The SNSB includes tests of language, visuospatial, memory, and frontal/executive functions. General cognition was evaluated using the MMSE. Dementia severity was measured using a global deterioration scale (GDS). Attention ability was evaluated using the digit span test (DST). Language function was based on the Korean version of the Boston naming test (K-BNT). Visuospatial function was based on the Rey complex figure test (RCFT) copy score. Verbal memory function was evaluated using the Seoul verbal learning test (SVLT) immediate recall, delayed recall, and recognition score. Visuospatial memory function was evaluated using the RCFT immediate recall, delayed recall, and recognition score. The frontal/executive function was calculated using the controlled oral word association test (COWAT) and Stroop test. Additionally, depressive symptoms and instrumental activities of daily living were evaluated using the Korean version of the short form geriatric depression scale and Seoul instrumental activity of daily living (S-IADL).

\section{Amyloid PET acquisition and measurement of amyloid deposition}

Participants underwent 18F-flutemetamol PET scans using a Discovery Ste/690 PET/CT scanner (GE, Milwaukee, WI, USA) with an identical protocol. 18F-flutemetamol was injected into the antecubital vein as a bolus with a mean dose of $185 \mathrm{MBq}$. After $90 \mathrm{~min}$, a $20 \mathrm{~min}$ PET scan (4×5 min dynamic frames) was performed. ${ }^{18} \mathrm{~F}$-flutemetamol PET scans were co-registered to individual MRI scans, which were normalized to a T1-weighted MRI template. Using transformation parameters, MRI co-registered ${ }^{18} \mathrm{~F}$-flutemetamol PET images were normalized to the MRI template. To quantify ${ }^{18} \mathrm{~F}$ flutemetamol retention, the standard uptake value ratio (SUVR) was obtained using the pons as a reference region. Global cortical $18 \mathrm{~F}$-flutemetamol retention was calculated from the volume-weighted average SUVR of bilateral ten cortical volumes of interest from the frontal, posterior cingulate, lateral temporal, parietal, and occipital lobes using the annotated anatomical labeling atlas. ${ }^{20}$ Based on a previous report on the elderly
Korean population and our observed data distribution, participants were considered to be positive for amyloid if their global cortical SUVR was greater than $0.634 .{ }^{21}$ Alternatively, we also evaluated amyloid positivity by a visual rating of experienced nuclear medicine specialists who were blinded to the clinical information of the participants.

\section{MRI acquisition and measurement of neurodegeneration}

MRI scan data were obtained using a 3.0T MR scanner. Structural MRI, including 3-dimension T1, T2, fluid-attenuated inversion recovery (FLAIR) imaging, was performed. All MR images were reviewed by neuroradiologists. Inevitably, MRI machines and detailed MRI parameters were slightly different between the clinical centers. These differences were thoroughly considered in the analytic process and are fully described in Supplementary Table 1 (in the online-only Data Supplement). In addition, from the subset of participants recruited from Ajou University Hospital, diffusion tensor imaging, susceptibility-weighted imaging, resting-state functional MRI (rs-fMRI), and arterial spin labeling imaging were also obtained. For measurement of neurodegeneration, visual rating of medial temporal lobe (MTL) atrophy in each hemisphere was evaluated by Schelten's criteria using T1 brain MR images, and then these two values were averaged. ${ }^{22}$ Alternatively, we also assessed neurodegeneration using voxel-based morphometry with diffeomorphic anatomical registration through an exponentiated lie algebra procedure on T1-weighted images. Preprocessing of the T1 brain MR data was performed using the Statistical Parametric Mapping software (SPM12; Wellcome Department of Cognitive Neurology, London, UK) implemented in MATLAB (Mathworks Inc., Natick, MA, USA). Before assessing gray matter density, we normalized each participant's image into the Montreal Neurological Institute space with the normalized images modulated to ensure that relative gray and white matter volumes were preserved following spatial normalization.

\section{Measurement of chronic CVD burden}

To measure chronic CVD burden, WMH were evaluated using the modified criteria of Fazekas et al. ${ }^{23}$ using FLAIR brain MR images. WMH was separately examined in the periventricular white matter and deep white matter lesions. The severity of deep white matter lesions was classified as mild (deep white matter $<10 \mathrm{~mm}$ ), moderate (deep white matter from 10 $\mathrm{mm}$ to $<25 \mathrm{~mm}$ ), and severe (deep white matter $>25 \mathrm{~mm}$ ) based on the greatest diameter of the lesions. The severity of periventricular white matter lesions was divided into mild (cap and band $<5 \mathrm{~mm}$ ), moderate (cap and band from $5 \mathrm{~mm}$ to $<10$ $\mathrm{mm}$ ), and severe (cap and band $>10 \mathrm{~mm}$ ), based on the size 
of the cap and band, which were perpendicular to the ventricle, respectively. The degree of overall WMH was assigned to one of three groups by using periventricular and deep white matter hyperintensity (PVWMH and DWMH) severity, such as mild (D1P1 and D1P2), moderate (neither mild nor severe; D1P3, D2P1, D2P2, D2P3, D3P1, and D3P2), and severe (D3P3). ${ }^{24}$

\section{Blood sampling and laboratory assessments}

For baseline routine laboratory tests and blood sampling for potential peripheral biomarker research, blood samples were taken after an overnight fast in the morning by venipuncture and collected in serum separating tubes and dipotassium ethylenediaminetetraacetic acid. Baseline routine blood laboratory tests included complete blood cell count, blood urea nitrates, creatinine, albumin, liver function tests, fasting serum glucose, glycated hemoglobin (HbA1c), serum lipids, total protein, folic acid, high-sensitivity C-reactive protein, fibrinogen, venereal disease research laboratory test, treponema pallidum hemagglutination, electrolyte analysis, vitamin B12 test, homocysteine, thyroid function test, and apolipoprotein $\mathrm{E}$ (APOE). Blood samples were stabilized and centrifuged at 3,000 rpm for $10 \mathrm{~min}$ at room temperature to obtain plasma and serum supernatants. To obtain samples with high purity, the plasma and serum supernatants were further centrifuged under the same conditions, collected, and immediately stored in a $-80^{\circ} \mathrm{C}$ deep freezer. Extracted genomic DNA specimens were also stored at $-80^{\circ} \mathrm{C}$ for future analyses.

\section{Single nucleotide polymorphism (SNP) genotyping arrays}

SNP genotyping was carried out using the Korea Biobank Array (Affymetrix Axiom KORV1.1-96 Array, Thermo Fisher Scientific, Santa Clara, CA, USA) at DNA Link Inc. (Seoul, Republic of Korea). ${ }^{25}$ For SNP quality control, SNPolisher was used to exclude low-quality markers by considering a poly high resolution, mono high resolution, and no minor homozygosity. In addition, SNPs with low call rates ( $<95 \%)$ or with a low p-value in the Hardy Weinberg Equilibrium test $\left(<10^{-6}\right)$ were excluded. For the sample quality controls, the following criteria were considered to provide better data: call rates $(\geq 97 \%)$, hetero rate (within \pm 2 ), multidimensional scaling (MDS) analysis (within coordinate \pm absolute value 0.02 ), singletons $(\leq 15)$, identity-by-state test, and gender mismatch. As a result, 769,894 SNPs with 949 participants were extracted for SNP genotyping.

\section{Measure of locomotor activity}

A subset of participants who were recruited from Ajou University Hospital were invited to wear a research-purposed accelerometer (Fitmeter; Fit. Life Inc., Suwon, Korea) on their non-dominant wrist for at least seven days while performing their usual activities at home. ${ }^{26,27}$ Activity counts in 1 min epochs from the first four consecutive days of data, starting at midnight, were analyzed to assess the rest-activity patterns. Some participants had irregular missing time on their data, indicating device removal or removal during their four-day assessment period. We used the imputation method "missing" to control for this, which was designed and validated for the accelerometer data imputation process using 2003-2004 National Health and Nutrition Examination Survey accelerometer data. ${ }^{28}$ Subsequently, we analyzed the mean activity with its variance and skewness using a classical statistical method. In addition, we assessed the rest-activity patterns and circadian phases using a nonparametric analysis method. Nonparametric analysis does not have a priori assumptions about daily activity patterns but instead extracts parameters based on raw activity counts. ${ }^{29}$

\section{Patient-derived dermal fibroblast}

To perform ex vivo research and the potential generation of induced pluripotent stem cells (iPSCs) harboring the same genetic background of participants, we cultured and established patient-derived dermal fibroblast cells. A subset of participants agreed and donated skin biopsies for patient-derived dermal fibroblast sampling. A skin biopsy was obtained within four weeks of the baseline assessment. We planned and modified our biopsy and culture protocol based on previous studies and our preliminary experiences. ${ }^{30-32}$ In brief, a skin biopsy $\left(3 \mathrm{~mm}^{3}\right)$ was taken from the upper inside arm under local anesthesia and aseptic conditions using a disposable punch. The tissue was immediately immersed in $5 \mathrm{~mL}$ of warmed culture medium and directly transferred to the laboratory at the same institution. Then, we placed the biopsy piece into a $100 \mathrm{~mm}$ dish and cut the tissue into small pieces with a scalpel with a small amount of medium to avoid drying. After 1 min of attachment, we added $10 \mathrm{~mL}$ of Dulbecco's modified Eagle medium (high glucose), supplemented with $20 \%$ fetal bovine serum and $1 \%$ penicillin/streptomycin. After one week, we changed the culture medium every two to three days. Once enough fibroblasts were identified around biopsy pieces, we trypsinized and passaged the $100 \mathrm{~mm}$ dish into another $100 \mathrm{~mm}$ dish for further processing (passage 1). Finally, we characterized and validated our fibroblast culture using the fibroblast marker protein SERPINH1 immunocytochemistry (Anti-SERPINH1 monoclonal antibody, Aviva, OACA06643).

\section{Statistical analysis}

Continuous variable data were reported as the mean and standard deviation (SD), and categorical data were reported as the number of participants and percentage. We used a one- 
way analysis of variance (ANOVA) for continuous variables and the chi-squared test for categorical variables for group comparisons. For subsequent subgroup comparisons, we applied the Tukey method. Statistical analyses were performed using R Statistical Software, version 3.5.3 (R Foundation for Statistical Computing, Vienna, Austria). Statistical significance was set at $\mathrm{p}<0.05$.

\section{RESULTS}

\section{Demographic characteristics of participants}

As of December 2020, a total of 1,013 participants were enrolled in BICWALZS. The demographic characteristics of the participants' group based on clinical diagnosis are described in Table 1. The mean (SD) age of participants was 72.8 (7.3) years, education years was 7.9 (4.8), depressive symptom score was 6.1 (4.9), and GDS score was 3.3 (1.0). Of all participants, $5 \%$ were female, $24 \%$ presented with diabetes, and $56 \%$ were diagnosed with hypertension. Approximately $28 \%$ of participants were apolipoprotein E (APOE) e4 allele carriers. For group comparison based on clinical diagnosis, we performed an ANOVA with subgroup post hoc tests using the Tukey method. Detailed group comparison results are presented in
Table 1. In brief, as expected, the AD and vascular dementia (VD) groups were older than the SCD or MCI groups. In addition, the $\mathrm{AD}$ and $\mathrm{VD}$ groups also exhibited more progressed disease features on the clinical dementia rating sum of the box score, MMSE score, and disability score by S-IADL. Finally, as expected, a higher proportion of the AD group were APOE e4 allele carriers compared to the SCD, MCI, or VD groups. We also assessed and described the demographic characteristics based on cognitive status and two neuroimaging biomarkers (Supplementary Table 2 in the online-only Data Supplement).

\section{MTL neurodegeneration, WMH, and cortical amyloid burden}

A total of 817 and 713 participants agreed and underwent brain MRI and amyloid PET, respectively. We first assessed MTL neurodegeneration using Schelten's (zero to four) scale, and a score of two or higher was considered moderate or above neurodegeneration. As a result, approximately $55.9 \%$ of the total participants who underwent brain MRI exhibited moderate or above MTL neurodegeneration. In detail, about 74.2\% of participants in the AD group had MTL neurodegeneration that was moderate or above. In contrast, $31.3 \%, 47.8 \%$, and $91.8 \%$ of participants showed moderate or greater MTL neu-

Table 1. Demographic characteristics of the study participants according to clinical diagnosis $(N=1,013)$

\begin{tabular}{|c|c|c|c|c|c|c|c|}
\hline $\begin{array}{c}\text { Participant } \\
\text { characteristics }\end{array}$ & $\begin{array}{c}\text { SCD } \\
(\mathrm{N}=168)\end{array}$ & $\begin{array}{c}\text { MCI } \\
(\mathrm{N}=534)\end{array}$ & $\begin{array}{c}\mathrm{AD} \\
(\mathrm{N}=211)\end{array}$ & $\begin{array}{c}\mathrm{VD} \\
(\mathrm{N}=80)\end{array}$ & $\begin{array}{l}\text { Others } \\
(\mathrm{N}=20)\end{array}$ & $\mathrm{p}^{\dagger}$ & $\mathrm{p}<0.05^{\ddagger}$ \\
\hline Age, years & $70.9 \pm 7.1$ & $72.6 \pm 6.9$ & $74.1 \pm 7.8$ & $75.1 \pm 7.0$ & $68.9 \pm 8.1$ & $<0.001$ & $a, b, c, d, e$ \\
\hline Female & $115(68.5)$ & $356(66.7)$ & $127(60.2)$ & $50(62.5)$ & $10(50.0)$ & 0.274 & \\
\hline Education, years & $7.7 \pm 4.0$ & $8.0 \pm 4.9$ & $8.5 \pm 5.0$ & $6.4 \pm 4.7$ & $8.8 \pm 4.6$ & 0.013 & $e, f$ \\
\hline Hypertension & $99(58.9)$ & $282(52.8)$ & $122(57.8)$ & $54(67.5)$ & $8(40.0)$ & 0.061 & \\
\hline Diabetes & $36(21.4)$ & $111(20.8)$ & $57(27.0)$ & $37(46.3)$ & $3(15.0)$ & $<0.001$ & \\
\hline Dyslipidemia & $66(39.3)$ & $202(37.8)$ & $58(27.5)$ & $29(36.3)$ & $5(25.0)$ & 0.042 & \\
\hline APOE e4 carrier & $26(15.5)$ & $133(24.9)$ & $98(46.5)$ & $17(21.3)$ & $4(20.0)$ & $<0.001$ & \\
\hline MMSE (0-30) & $26.2 \pm 2.8$ & $24.2 \pm 3.8$ & $19.0 \pm 5.1$ & $18.2 \pm 4.8$ & $20.6 \pm 5.3$ & $<0.001$ & $\mathrm{a}, \mathrm{b}, \mathrm{c}, \mathrm{d}, \mathrm{e}$ \\
\hline S-IADL & $3.4 \pm 3.6$ & $6.6 \pm 5.6$ & $16.4 \pm 10.4$ & $19.7 \pm 11.1$ & $19.1 \pm 9.2$ & $<0.001$ & $a, b, c, d, e, f$ \\
\hline SGDS-K & $4.3 \pm 4.0$ & $6.6 \pm 5.0$ & $5.4 \pm 4.6$ & $6.9 \pm 5.1$ & $9.9 \pm 4.1$ & $<0.001$ & $\mathrm{a}, \mathrm{c}, \mathrm{d}$ \\
\hline GDS & $2.3 \pm 0.5$ & $3.0 \pm 0.7$ & $4.2 \pm 0.9$ & $4.3 \pm 0.9$ & $4.4 \pm 1.1$ & $<0.001$ & \\
\hline $\mathrm{A} \beta$ deposition on PET & $\mathrm{N}=82$ & $\mathrm{~N}=400$ & $\mathrm{~N}=152$ & $\mathrm{~N}=62$ & $\mathrm{~N}=17$ & $<0.001$ & \\
\hline$(\mathrm{N}=713)$ & $7(8.5)$ & $107(26.8)$ & $129(84.9)$ & $9(14.5)$ & $2(11.8)$ & & \\
\hline Schelten's scale & $1.2 \pm 0.8$ & $1.6 \pm 0.8$ & $2.3 \pm 0.9$ & $2.6 \pm 0.8$ & $1.9 \pm 0.7$ & $<0.001$ & $a, b, c, d, e, f$ \\
\hline WMH on MRI $(\mathrm{N}=817)$ & $\mathrm{N}=102$ & $\mathrm{~N}=441$ & $\mathrm{~N}=182$ & $\mathrm{~N}=73$ & $\mathrm{~N}=19$ & $<0.001$ & \\
\hline Minimal & $73 \pm 71.6$ & $283(64.2)$ & $94(51.7)$ & $10(13.7)$ & $16(84.2)$ & & \\
\hline Moderate & $25(24.5)$ & $139(31.5)$ & $74(40.7)$ & $33(45.2)$ & $3(15.8)$ & & \\
\hline Severe & $4(3.9)$ & $19(4.3)$ & $14(7.7)$ & $30(41.1)$ & $0(0.0)$ & & \\
\hline
\end{tabular}

Data are shown as the mean \pm SD or number (\%). ${ }^{\dagger}$ analysis of variance or chi-square test; $\ddagger$ tukey post hoc analysis. a, SCD vs. MCI; b, SCD vs. $\mathrm{AD} ; \mathrm{c}, \mathrm{SDC}$ vs. VD; d, MCI vs. AD; e, MCI vs. VD; f, AD vs. VD. SCD, subjective cognitive decline; MCI, mild cognitive impairment; AD, Alzheimer's dementia; VD, vascular dementia; APOE, apolipoprotein E; MMSE, mini-mental state examination; S-IADL, Seoul instrumental activities of daily living; SGDS-K, Korean version of the short form of geriatric depression scale; GDS, global deterioration scale; WMH, white matter hyperintensities 
Table 2. Neuropsychological test results according to clinical diagnosis $(\mathrm{N}=1,013)$

\begin{tabular}{|c|c|c|c|c|c|c|c|}
\hline Neuropsychological test & $\begin{array}{c}\text { SCD } \\
(\mathrm{N}=168)\end{array}$ & $\begin{array}{c}\text { MCI } \\
(\mathrm{N}=534)\end{array}$ & $\begin{array}{c}\mathrm{AD} \\
(\mathrm{N}=211)\end{array}$ & $\begin{array}{c}\mathrm{VD} \\
(\mathrm{N}=80)\end{array}$ & $\begin{array}{l}\text { Others } \\
(\mathrm{N}=20)\end{array}$ & $\mathrm{p}^{\dagger}$ & $\mathrm{p}<0.05^{\ddagger}$ \\
\hline \multicolumn{8}{|l|}{ Attention function } \\
\hline Digit Span Test-backward & $-0.14(1.11)$ & $-0.51(1.09)$ & $-1.09(1.29)$ & $-1.27(1.23)$ & $-1.38(1.40)$ & $<0.001$ & $\mathrm{a}, \mathrm{b}, \mathrm{c}, \mathrm{d}, \mathrm{e}$ \\
\hline \multicolumn{8}{|l|}{ Language function } \\
\hline Boston naming test & $0.40(0.85)$ & $-0.47(1.32)$ & $-1.61(1.65)$ & $-1.60(1.97)$ & $-2.35(2.18)$ & $<0.001$ & $\mathrm{a}, \mathrm{b}, \mathrm{c}, \mathrm{d}, \mathrm{e}$ \\
\hline \multicolumn{8}{|l|}{ Visuospatioal function } \\
\hline RCFT-copy & $-0.04(1.07)$ & $-0.70(4.70)$ & $-2.34(2.48)$ & $-2.50(2.02)$ & $-3.38(3.07)$ & $<0.001$ & $b, c, d, e$ \\
\hline \multicolumn{8}{|l|}{ Memory function } \\
\hline SVLT-delayed recall & $0.21(0.95)$ & $-0.89(1.08)$ & $-1.84(0.94)$ & $-1.64(0.86)$ & $-1.88(0.72)$ & $<0.001$ & $\mathrm{a}, \mathrm{b}, \mathrm{c}, \mathrm{d}, \mathrm{e}$ \\
\hline RCFT-delayed recall & $0.13(0.98)$ & $-0.73(0.96)$ & $-1.59(0.78)$ & $-1.16(0.80)$ & $-1.12(1.13)$ & $<0.001$ & $a, b, c, d, e, f$ \\
\hline \multicolumn{8}{|l|}{ Frontal/executive function } \\
\hline Stroop test-color reading & $0.09(0.95)$ & $-0.77(1.28)$ & $-1.82(1.63)$ & $-2.10(1.24)$ & $-2.74(1.53)$ & $<0.001$ & $\mathrm{a}, \mathrm{b}, \mathrm{c}, \mathrm{d}, \mathrm{e}$ \\
\hline COWAT-phonemic task & $0.06(0.89)$ & $-0.64(0.92)$ & $-1.36(1.05)$ & $-1.58(0.73)$ & $-1.56(0.83)$ & $<0.001$ & $\mathrm{a}, \mathrm{b}, \mathrm{c}, \mathrm{d}, \mathrm{e}$ \\
\hline
\end{tabular}

†analysis of variance; †tukey post hoc analysis. a, SCD vs. MCI; b, SCD vs. AD; c, SDC vs. VD; d, MCI vs. AD; e, MCI vs. VD; f, AD vs. VD. $\mathrm{AD}$, Alzheimer's dementia; MCI, mild cognitive impairment; SCD, subjective cognitive decline; VD, vascular dementia; RCFT, Rey complex figure test; SVLT, Seoul verbal learning test; COWAT, controlled oral word association test-phonemic task

rodegeneration in the SCD, MCI, and VD groups, respectively (Table 1). In addition, we assessed WMH using the modified criteria of Fazekas et al., ${ }^{23}$ and participants in the VD group had more severe WMH in their deep white matter and periventricular regions (Table 1). We also analyzed amyloid PET and found that $35.6 \%$ of participants had 0.634 or higher SUVR on their amyloid PET, suggesting problematic cortical amyloid deposition. In detail, about $84.9 \%$ of participants in the $\mathrm{AD}$ group exhibited amyloid positivity on imaging. In contrast, $8.5 \%, 26.8 \%$, and $14.5 \%$ of participants showed amyloid positivity in the SCD, MCI, and VD groups, respectively. We also assessed and described MTL neurodegeneration, WMH, and cortical amyloid burden based on cognitive status and two neuroimaging biomarkers (Supplementary Table 2 in the online-only Data Supplement).

\section{Neuropsychological test}

We performed neuropsychological tests to assess the multidomain cognitive function of the study participants and compared the results of SCD, MCI, AD, and VD groups (Table 2). The detailed results are presented in Table 2. In summary, DST-backward scores (attention), K-BNT scores (language), RCFT-copy (visuospatial function), SVLT-delayed recall (verbal memory), RCFT-delayed recall (visuospatial memory), Stroop test (frontal/executive function), and COWAT (frontal/executive function) were significantly different between all groups, except between the $\mathrm{AD}$ and $\mathrm{VD}$ groups $(\mathrm{SCD}>\mathrm{MCI}>$ $\mathrm{AD}$ or $\mathrm{VD}, \mathrm{p}<0.001$ for all five tests). In addition, the RCFTdelayed recall score representing visuospatial memory function was different between all four groups $(\mathrm{SCD}>\mathrm{MCI}>\mathrm{VD}>\mathrm{AD}$,

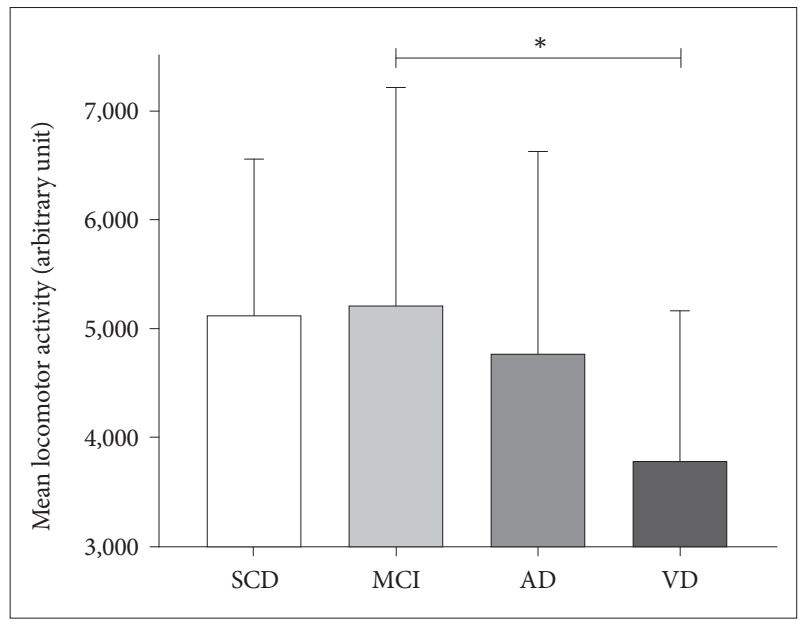

Figure 1. Mean locomotor activity assessed by actigraphy according to clinical diagnosis. ${ }^{*} \mathrm{p}<0.05$ on Tukey post hoc analysis. AD, Alzheimer's dementia; $\mathrm{MCl}$, mild cognitive impairment; SCD, subjective cognitive decline; VD, vascular dementia.

$\mathrm{p}<0.001)$. We also assessed the neuropsychological test results based on cognitive status and two neuroimaging biomarkers (Supplementary Table 3 in the online-only Data Supplement).

\section{Locomotor activity}

Using actigraphy, activity counts in $1 \mathrm{~min}$ epochs from the first four consecutive days of data were analyzed to assess the locomotor activity of the participants. We assessed the group differences in mean locomotor activity with skewness. Significant differences in the mean activity were noted between the $\mathrm{MCI}$ and VD groups (mean \pm SD, $5122 \pm 1433$ vs. $3775 \pm 1384$, respectively, $\mathrm{p}=0.02$ ) (Figure 1). However, the skewness did not 


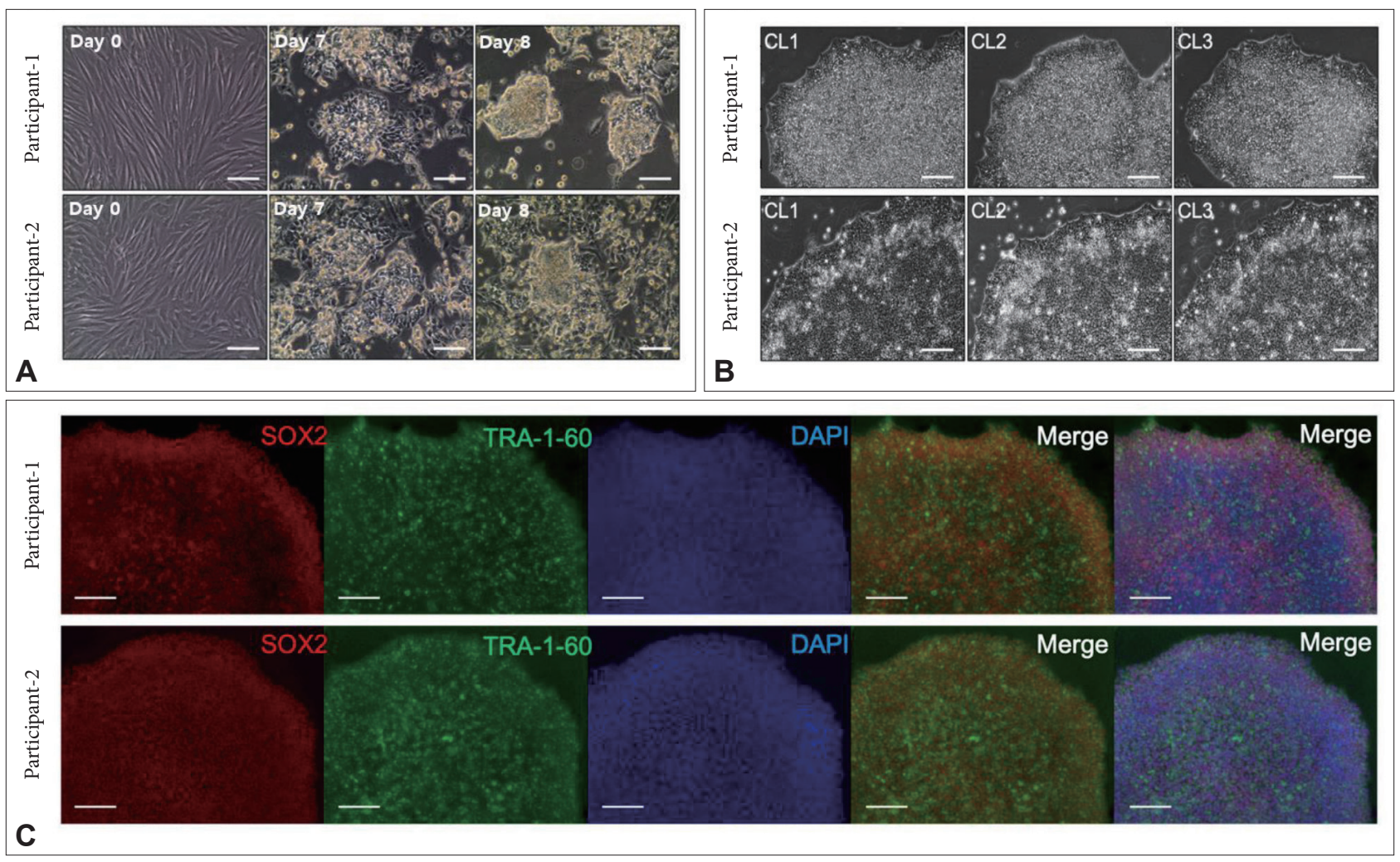

Figure 2. Induced pluripotent stem cells generated from patient-derived dermal fibroblast of BICWALZS participants. A: Bright imaging morphology of patient-derived dermal fibroblast and reprogramming process by conventional technique using Sendai virus; scale bars indicate $200 \mu \mathrm{m}$. B: Bright imaging morphology of induced pluripotent stem cell colonies, six clones from two participants, scale bars indicate $200 \mu \mathrm{m}$. C: Representative images of SOX2 and TRA-1-60 expression (immunofluorescence) in iPSC colonies. CL, clone; DAPI, 4,6-diamidino-2-phenylindole; SOX2, SRY-box transcription factor 2; TRA-1-60, podocalyxin.

have a group difference. In addition, the mean activity and skewness showed a large variance between participants. Considering this large variance, locomotor activity should be analyzed with potential confounders, such as sex, body mass index, and comorbidities. In addition, various characteristics such as rest-activity patterns or circadian phase need to be considered and analyzed with clinical characteristics and neuroimaging biomarkers such as disability, cognitive function, cortical amyloid burden, and MTL neurodegeneration.

\section{Patient-derived dermal fibroblast}

For the purposes of possible ex vivo research and potential generation of iPSCs or brain organoids harboring the same genetic background of participants, we performed skin punch biopsy and retained patient-derived dermal fibroblasts. Stabilized patient-derived dermal fibroblasts exhibited a typical cylindrical morphology with good proliferative potential (Figure 2). As a preliminary experiment, we generated six clones of iPSCs from two derived dermal fibroblasts using the conventional technique with the Sendai virus and checked the typical morphology of iPSCs with basic marker proteins for iPSCs (SOX2 and TRA-1-60). Finally, we performed karyotypic anal- ysis, and there were no chromosomal abnormalities or loss of genetic integrity.

\section{DISCUSSION}

Considering the multifactorial nature of neurodegenerative disorders and their heterogeneous clinical presentations, gathering, and integration of multi-layered clinical information, biospecimens, and patient-derived characteristics are critical steps for finding new biomarkers and developing disease modeling processes. In the BICWALZS, we recruited 1,013 older adults who complained of cognitive decline and established a multi-layered dataset including demographic, peripheral bloodbased, neuropsychological, and neuroimaging variables of the study participants. In addition, we performed SNP genotyping arrays, locomotor activity assessments and established patient-derived dermal fibroblasts in a subset of participants. We hope that our integrated clinical data structure, biospecimen, genome-wide genetic information, and patient-derived cells will facilitate optimal biomedical research for neurodegenerative disorders and disease modeling processes not only in South Korea but also abroad. 
Characterization of participants' demographic, neuropsychological, and neuroimaging results compared to similarly designed dementia cohorts might help identify and consider the cohort characteristics and possible selection bias of BICWALZS. For this, we compared our participant characteristics (age, sex, education year, MMSE score, APOE e4 allele carrier, and amyloid positivity on PET) with meta-analysis results assessing 29 cohorts, including 1,897 dementia patients and 1,849 control participants. ${ }^{33}$ As a result, AD participants in BICWALZS were slightly older than previous meta-analysis patients with $\mathrm{AD}$ (mean $\pm \mathrm{SD}, 74.1 \pm 7.8$ and 69.4 \pm 9.3 years, $\mathrm{p}<$ 0.001). However, the age of VD participants in BICWALZS was similar to the age of meta-analysis VD patients (mean \pm $\mathrm{SD}, 75.1 \pm 7.0$ and $74.5 \pm 8.5$ years, respectively, $\mathrm{p}=0.57$ ). We also noted that $\mathrm{AD}$ participants in BICWALZS were less educated than meta-analysis patients with $\mathrm{AD}$ (mean $\pm \mathrm{SD}, 8.4 \pm 5.0$ and $13.8 \pm 3.6$ years, $\mathrm{p}<0.001$ ). The education difference of the VD group showed a similar pattern (mean $\pm \mathrm{SD}, 6.4 \pm 4.7$ and $10.1 \pm$ 4.2 years, respectively, $\mathrm{p}<0.001$ ). We believe that the difference in education years reflects cultural differences and may have resulted in slightly lower MMSE scores of the participants with $\mathrm{AD}$ or VD in BICWALZS compared to the other meta-analysis patients (data not shown).

We also compared the two most well-known AD biomarkers, the proportion of APOE e4 allele carriers and amyloid positivity on PET. In BICWALZS participants, $46.4 \%$ of $\mathrm{AD}$ participants and $21.2 \%$ of VD participants were APOE e 4 allele carriers. Meta-analysis results showed a higher proportion of APOE e4 allele carriers in patients with AD and VD but a relatively preserved $\mathrm{e} 4$ allele carrier ratio between patients with $\mathrm{AD}$ and $\mathrm{VD}$ (61.1\% in $\mathrm{AD}$ and $28.0 \%$ in $\mathrm{VD})$. This allele frequency difference was consistent with previous studies that reported a relatively lower APOE e4 allele frequency in Asian populations. ${ }^{34-36}$ Concerning amyloid positivity, we noted that the proportion of amyloid-positive participants was similar in BICWALZS AD participants and meta-analysis patients with $\mathrm{AD}(85.4 \%$ and $87.8 \%$, respectively, $\mathrm{p}=0.43)$. Similarly, the proportion of amyloid positivity in the VD group was not significantly different between BICWALZS VD participants and meta-analysis patients with VD (34.6\% and 30.4\%, respectively; $\mathrm{p}=0.58$ ). Taken together, although BICWALZS AD participants were slightly older than the mean age of $\mathrm{AD}$ patients in 26 dementia cohorts, other participant characteristics such as higher education years, a higher proportion of APOE e4 allele carriers, and higher amyloid positivity in $\mathrm{AD}$ participants were generally similar to those of other dementia cohort participants. Finally, we also compared participants' characteristics of MCI in BICWALZS with an independent nationwide cohort in Korea called KBASE-V. ${ }^{21}$ In summary, MCI participants in BICWALZS had similar age and education years as
MCI participants in KBASE-V ( $\mathrm{p}=0.63$ for age, $\mathrm{p}=0.09$ for education years). The proportion of APOE e 4 carriers and amyloid positivity in MCI participants was similar between the BICWALZS and KBASE-V cohorts ( $\mathrm{p}=0.61$ for APOE e4 carrier, and $\mathrm{p}=0.10$ for amyloid positivity). Thus, we think that the baseline characteristics of the BICWALZS participants in the MCI, AD, and VD groups are generally acceptable and consistent with worldwide dementia cohorts and independent cohorts in Korea.

Assessing locomotor activity by actigraphy in BICWALZS participants was based on our hypothesis about a possible association between rest-activity patterns, circadian phase, and neurodegeneration in older adults. Although there is controversy on the causality of circadian disruption on neurodegeneration, several previous studies, including our report, suggest a possible association between rest-activity patterns or circadian phase changes with neurodegeneration in patients with cognitive decline. ${ }^{37,38}$ A recent report also suggested a possible association between rest-activity patterns, white matter degeneration, and clinical risk factors in individuals at risk for dementia. ${ }^{39}$ Integrated analysis assessing the possible association of circadian rest-activity pattern changes with multiple neuroimaging biomarkers (gray matter density, white matter microstructure, resting-status functional connectivity, perfusion, and amyloid deposition in the cortex or subcortex) are in progress in our laboratory and co-work research teams.

Patient-derived dermal fibroblasts are invaluable biospecimens that have great potential for ex vivo research and disease modeling using iPSCs or brain organoid generation..$^{15,40,41}$ One of our research teams is performing ex vivo research using patient-derived dermal fibroblasts to assess the cellular circadian rhythm of BICWALZS participants. In this biochemical experiment, we transfected a lentiviral vector (pABpuro-BluF) into patient-derived dermal fibroblasts and successfully measured Bmal1 transcriptional activity using a real-time luminometer. ${ }^{42}$ In addition, we successfully generated six clones of iPSCs from two participants and identified two iPSC marker proteins and normal karyotypic properties. We hope that the patient-derived dermal fibroblasts of BICWALZS participants will facilitate optimal biochemical and disease modeling research in the neurodegenerative research field.

In conclusion, we constructed a BICWALZS cohort including patients with SCD, MCI, AD, and VD. The baseline characteristics of the BICWALZS participants in the MCI, AD, and VD groups are generally acceptable and consistent with worldwide dementia cohorts and independent cohorts in Korea. ${ }^{21,33}$ We gathered demographic, neuropsychological, and neuroimaging variables as well as genome-wide SNP genotyping arrays, locomotor activity assessment using actigraphy, and patient-derived dermal fibroblast sampling. We hope that 
our integrated and multi-layered data structure and invaluable biospecimens will facilitate optimal biomedical research for neurodegenerative disorders and disease modeling experiments.

\section{Supplementary Materials}

The online-only Data Supplement is available with this article at https://doi.org/10.30773/pi.2021.0335.

\section{Availability of Data and Material}

The datasets generated or analyzed during the study are available from the corresponding authors upon reasonable request. For access and sharing, biological resources of the BICWALZS were stored in the BICWALZS consortium biobank (www.bicwalzs.com/) as well as the National Biobank of Korea (www.nih.go.kr/biobank) which is a central biobank supporting for the Korea Biobank Project.

\section{Conflicts of Interest}

The authors have no potential conflicts of interest to disclose.

\section{Author Contributions}

Conceptualization: Sang Joon Son, Chang Hyung Hong. Data curation: Hyun Woong Roh, Na-Rae Kim, Dong-gi Lee. Formal analysis: Hyun Woong Roh, Na-Rae Kim, Dong-gi Lee. Investigation: Hyun Woong Roh, Jae-Youn Cheong, Sang Won Seo, Seong Hye Choi, Eun-Joo Kim, Soo Hyun Cho, Byeong C. Kim, Seong Yoon Kim, Sang Joon Son, Chang Hyung Hong. Methodology: Eun Young Kim, Jaerak Chang, Sang Yoon Lee, Dukyong Yoon, Jin Wook Choi, Young-Sil An, Hee Young Kang, Hyunjung Shin, Bumhee Park. Resources: Sang Joon Son, Chang Hyung Hong. Software: Hyun Woong Roh, Na-Rae Kim, Bumhee Park. Supervision: Sang Joon Son, Chang Hyung Hong. Validation: Sang Joon Son, Chang Hyung Hong. Visualization: Hyun Woong Roh, Na-Rae Kim. Writing_original draft: Hyun Woong Roh, Na-Rae Kim. Writing_-review \& editing: Bumhee Park, Sang Joon Son, Chang Hyung Hong.

\section{ORCID iDs}

Hyun Woong Roh
Na-Rae Kim
Dong-gi Lee
Jae-Youn Cheong
Sang Won Seo
Seong Hye Choi
Eun-Joo Kim
Soo Hyun Cho
Byeong C. Kim
Seong Yoon Kim
Eun Young Kim
Jaerak Chang
Sang Yoon Lee
Dukyong Yoon
Jin Wook Choi
Young-Sil An
Hee Young Kang
Hyunjung Shin
Bumhee Park
Sang Joon Son
Chang Hyung Hong

https://orcid.org/0000-0002-1333-358X https://orcid.org/0000-0002-1321-2155 https://orcid.org/0000-0002-7885-7894 https://orcid.org/0000-0001-6246-1783 https://orcid.org/0000-0002-8747-0122 https://orcid.org/0000-0002-0680-8364 https://orcid.org/0000-0002-8460-1377 https://orcid.org/0000-0002-4262-1468 https://orcid.org/0000-0001-6827-6730 https://orcid.org/0000-0002-1077-6289 https://orcid.org/0000-0001-6466-8622 https://orcid.org/0000-0003-1732-2076 https://orcid.org/0000-0003-4695-0266 https://orcid.org/0000-0003-1635-8376 https://orcid.org/0000-0002-2396-4705 https://orcid.org/0000-0003-0777-6226 https://orcid.org/0000-0001-8697-4292 https://orcid.org/0000-0001-8347-8277 https://orcid.org/0000-0002-5271-1571 https://orcid.org/0000-0001-7434-7996 https://orcid.org/0000-0003-3258-7611

\section{Funding Statement}

This study was conducted with biospecimens and data from the consortium of the Biobank Innovations for Chronic Cerebrovascular Disease with ALZheimer's Disease Study (BICWALZS), which was funded by the Korea Disease Control and Prevention Agency for the Korea Biobank
Project (\#4845-303). This work was also supported and funded by the grant from National Research Foundation of Korea (NRF), funded by the Ministry of Science and ICT (NRF-2019R1A5A2026045) and the grant from the Korea Health Technology R\&D Project through the Korea Health Industry Development Institute (KHIDI), and the Ministry of Health \& Welfare, Republic of Korea (grant number: HR21C1003).

\section{REFERENCES}

1. Winblad B, Amouyel P, Andrieu S, Ballard C, Brayne C, Brodaty H, et al. Defeating Alzheimer's disease and other dementias: a priority for European science and society. Lancet Neurol 2016;15:455-532.

2. Hardy J, De Strooper B. Alzheimer's disease: where next for anti-amyloid therapies? Brain 2017;140:853-855.

3. Doody RS, Thomas RG, Farlow M, Iwatsubo T, Vellas B, Joffe S, et al. Phase 3 trials of solanezumab for mild-to-moderate Alzheimer's disease. N Engl J Med 2014;370:311-321.

4. Schneider L. A resurrection of aducanumab for Alzheimer's disease. Lancet Neurol 2020;19:111-112.

5. Kapasi A, DeCarli C, Schneider JA. Impact of multiple pathologies on the threshold for clinically overt dementia. Acta Neuropathol 2017;134: 171-186.

6. Brenowitz WD, Hubbard RA, Keene CD, Hawes SE, Longstreth WT Jr, Woltjer RL, et al. Mixed neuropathologies and estimated rates of clinical progression in a large autopsy sample. Alzheimers Dement 2017;13: 654-662.

7. Dichgans M, Leys D. Vascular cognitive impairment. Circ Res 2017;120: 573-591.

8. van der Flier WM, Skoog I, Schneider JA, Pantoni L, Mok V, Chen CLH, et al. Vascular cognitive impairment. Nat Rev Dis Primers 2018;4:18003.

9. Ye BS, Seo SW, Kim JH, Kim GH, Cho H, Noh Y, et al. Effects of amyloid and vascular markers on cognitive decline in subcortical vascular dementia. Neurology 2015;85:1687-1693.

10. Chui HC, Zarow C, Mack WJ, Ellis WG, Zheng L, Jagust WJ, et al. Cognitive impact of subcortical vascular and Alzheimer's disease pathology. Ann Neurol 2006;60:677-687.

11. Lee MJ, Seo SW, Na DL, Kim C, Park JH, Kim GH, et al. Synergistic effects of ischemia and beta-amyloid burden on cognitive decline in patients with subcortical vascular mild cognitive impairment. JAMA Psychiatry 2014;71:412-422.

12. Chong JSX, Jang H, Kim HJ, Ng KK, Na DL, Lee JH, et al. Amyloid and cerebrovascular burden divergently influence brain functional network changes over time. Neurology 2019;93:e1514-e1525.

13. Palmqvist S, Tideman P, Cullen N, Zetterberg H, Blennow K, Alzheimer's Disease Neuroimaging Initiative, et al. Prediction of future Alzheimer's disease dementia using plasma phospho-tau combined with other accessible measures. Nat Med 2021;27:1034-1042.

14. Penney J, Ralvenius WT, Tsai LH. Modeling Alzheimer's disease with iPSC-derived brain cells. Mol Psychiatry 2020;25:148-167.

15. Rivetti di Val Cervo P, Besusso D, Conforti P, Cattaneo E. hiPSCs for predictive modelling of neurodegenerative diseases: dreaming the possible. Nat Rev Neurol 2021;17:381-392.

16. Winblad B, Palmer K, Kivipelto M, Jelic V, Fratiglioni L, Wahlund LO, et al. Mild cognitive impairment--beyond controversies, towards a consensus: report of the International Working Group on Mild Cognitive Impairment. J Intern Med 2004;256:240-246.

17. McKhann GM, Knopman DS, Chertkow H, Hyman BT, Jack CR Jr, Kawas $\mathrm{CH}$, et al. The diagnosis of dementia due to Alzheimer's disease: recommendations from the National Institute on Aging-Alzheimer's Association workgroups on diagnostic guidelines for Alzheimer's disease. Alzheimers Dement 2011;7:263-269.

18. Sachdev PS, Blacker D, Blazer DG, Ganguli M, Jeste DV, Paulsen JS, et al. Classifying neurocognitive disorders: the DSM-5 approach. Nat Rev Neurol 2014;10:634-642.

19. Ahn HJ, Chin J, Park A, Lee BH, Suh MK, Seo SW, et al. Seoul Neuro- 
psychological Screening Battery-dementia version (SNSB-D): a useful tool for assessing and monitoring cognitive impairments in dementia patients. J Korean Med Sci 2010;25:1071-1076.

20. Tzourio-Mazoyer N, Landeau B, Papathanassiou D, Crivello F, Etard O, Delcroix N, et al. Automated anatomical labeling of activations in SPM using a macroscopic anatomical parcellation of the MNI MRI singlesubject brain. Neuroimage 2002;15:273-289.

21. Hwang J, Jeong JH, Yoon SJ, Park KW, Kim EJ, Yoon B, et al. Clinical and biomarker characteristics according to clinical spectrum of Alzheimer's Disease $(\mathrm{AD})$ in the validation cohort of Korean brain aging study for the early diagnosis and prediction of AD. J Clin Med 2019;8: 341 .

22. Scheltens P, Barkhof F, Leys D, Pruvo JP, Nauta JJ, Vermersch P, et al. A semiquantative rating scale for the assessment of signal hyperintensities on magnetic resonance imaging. J Neurol Sci 1993;114:7-12.

23. Fazekas F, Chawluk JB, Alavi A, Hurtig HI, Zimmerman RA. MR signal abnormalities at $1.5 \mathrm{~T}$ in Alzheimer's dementia and normal aging. AJR Am J Roentgenol 1987;149:351-356.

24. Noh Y, Lee Y, Seo SW, Jeong JH, Choi SH, Back JH, et al. A new classification system for ischemia using a combination of deep and periventricular white matter hyperintensities. J Stroke Cerebrovasc Dis 2014; 23:636-642.

25. Moon S, Kim YJ, Han S, Hwang MY, Shin DM, Park MY, et al. The Korea Biobank Array: Design and Identification of Coding Variants Associated with Blood Biochemical Traits. Sci Rep 2019;9:1382.

26. Kim DY, Jung YS, Park RW, Joo NS. Different location of triaxial accelerometer and different energy expenditures. Yonsei Med J 2014;55:11451151.

27. Shin DW, Yun JM, Shin JH, Kwon H, Min HY, Joh HK, et al. Enhancing physical activity and reducing obesity through smartcare and financial incentives: a pilot randomized trial. Obesity (Silver Spring) 2017;25: 302-310.

28. Lee JA, Gill J. Missing value imputation for physical activity data measured by accelerometer. Stat Methods Med Res 2018;27:490-506.

29. Witting W, Kwa IH, Eikelenboom P, Mirmiran M, Swaab DF. Alterations in the circadian rest-activity rhythm in aging and Alzheimer's disease. Biol Psychiatry 1990;27:563-572.

30. Takashima A. Establishment of fibroblast cultures. Curr Protoc Cell Biol 2001; Chapter 2: Unit 21.
31. Vangipuram M, Ting D, Kim S, Diaz R, Schule B. Skin punch biopsy explant culture for derivation of primary human fibroblasts. J Vis Exp 2013;(77):e3779.

32. Yang S, Van Dongen HP, Wang K, Berrettini W, Bucan M. Assessment of circadian function in fibroblasts of patients with bipolar disorder. Mol Psychiatry 2009;14:143-155.

33. Ossenkoppele R, Jansen WJ, Rabinovici GD, Knol DL, van der Flier WM, van Berckel BN, et al. Prevalence of amyloid PET positivity in dementia syndromes: a meta-analysis. JAMA 2015;313:1939-1949.

34. Corbo RM, Scacchi R. Apolipoprotein E (APOE) allele distribution in the world. Is APOE*4 a 'thrifty' allele? Ann Hum Genet 1999;63:301310.

35. Crean S, Ward A, Mercaldi CJ, Collins JM, Cook MN, Baker NL, et al. Apolipoprotein E epsilon4 prevalence in Alzheimer's disease patients varies across global populations: a systematic literature review and meta-analysis. Dement Geriatr Cogn Disord 2011;31:20-30.

36. Ward A, Crean S, Mercaldi CJ, Collins JM, Boyd D, Cook MN, et al. Prevalence of apolipoprotein E4 genotype and homozygotes (APOE e4/4) among patients diagnosed with Alzheimer's disease: a systematic review and meta-analysis. Neuroepidemiology 2012;38:1-17.

37. Leng Y, Musiek ES, Hu K, Cappuccio FP, Yaffe K. Association between circadian rhythms and neurodegenerative diseases. Lancet Neurol 2019; 18:307-318.

38. Roh HW, Choi JG, Kim NR, Choe YS, Choi JW, Cho SM, et al. Associations of rest-activity patterns with amyloid burden, medial temporal lobe atrophy, and cognitive impairment. EBioMedicine 2020;58:102881.

39. Palmer JR, Duffy SL, Meares S, Pye J, Calamante F, Cespedes M, et al. Rest-activity functioning is related to white matter microarchitecture and modifiable risk factors in older adults at-risk for dementia. Sleep 2021;44:zsab007.

40. Koo B, Choi B, Park H, Yoon KJ. Past, present, and future of brain organoid technology. Mol Cells 2019;42:617-627.

41. Arber C, Alatza A, Leckey CA, Paterson RW, Zetterberg H, Wray S. Mass spectrometry analysis of tau and amyloid-beta in iPSC-derived models of Alzheimer's disease and dementia. J Neurochem 2021;159:305-317.

42. Brown SA, Fleury-Olela F, Nagoshi E, Hauser C, Juge C, Meier CA, et al. The period length of fibroblast circadian gene expression varies widely among human individuals. PLoS Biol 2005;3:e338. 
Supplementary Table 1. Brain MRI and amyloid PET technical parameters according to clinical sites

\begin{tabular}{|c|c|c|c|c|c|c|c|}
\hline MRI & $\mathrm{A}[\mathrm{GE}]$ & B [Philips] & $\mathrm{C}$ [GE] & $\mathrm{D}[\mathrm{GE}]$ & E [Siemens] & F [GE] & G [Philips] \\
\hline T1 3D & $\begin{array}{l}256-512 \times 256-512 \times 140-182 \\
(0.391-0.859,0.391-0.859,1) \\
{[\mathrm{TR}=7.1-9.9 \mathrm{~s}, \mathrm{TE}=2.84-4.8 \mathrm{~ms}]}\end{array}$ & $\begin{array}{c}80-512 \times 480-512 \times 360(0.5,0.5,0.5) \\
{[\mathrm{TR}=9.758-9.910 \mathrm{~s}, \mathrm{TE}=4.588-4.605 \mathrm{~ms}]}\end{array}$ & $\begin{array}{l}256 \times 256 \times 148-180 \\
(0.922-1.016,0.922-1.016,1.2) \\
{[\mathrm{TR}=7.4-7.644 \mathrm{~s}, \mathrm{TE}=2.708-2.788 \mathrm{~ms}]}\end{array}$ & $\begin{array}{l}256 \times 256 \times 152-196 \\
(0.7813-0.9375,0.7813-0.9375,1) \\
{[T R=7.472-8.644 \mathrm{~s}, \mathrm{TE}=2.752-3.288 \mathrm{~ms}]}\end{array}$ & $\begin{array}{c}256 \times 256 \times 256(0.977,0.977) \\
{[\mathrm{TR}=1.8 \mathrm{~s}, \mathrm{TE}=2.07 \mathrm{~ms}]}\end{array}$ & $\begin{array}{c}512 \times 512 \times 118-144(0.4688,0.4688,1.3) \\
{[\mathrm{TR}=8.172-13.98 \mathrm{~s}, \mathrm{TE}=3.236-6.008 \mathrm{~ms}]}\end{array}$ & $\begin{array}{l}480 \times 480 \times 360(0.5,0.5,0.5) \\
{[\mathrm{TR}=9.83-9.908 \mathrm{~s}, \mathrm{TE}=4.59-4.605 \mathrm{~ms}]}\end{array}$ \\
\hline T2_FLAIR & $\begin{array}{l}512 \times 512 \times 28-32(0.391,0.391,5) \\
{[\mathrm{TR}=8.8-10 \mathrm{~s}, \mathrm{TE}=86.152-128.16 \mathrm{~ms}]}\end{array}$ & $\begin{array}{l}521-560 \times 512-560 \times 80 \\
(0.446-0.469,0.446-0.469,2) \\
{[\text { TR }=11 \mathrm{~s}, \mathrm{TE}=125 \mathrm{~ms}]}\end{array}$ & $\begin{array}{l}256-512 \times 256-512 \times 23-75 \\
(0.430-0.938,0.430-0.938,2-6.5) \\
{[\mathrm{TR}=9 \mathrm{~s}, \mathrm{TE}=80.682-146.944 \mathrm{~ms}]}\end{array}$ & $\begin{array}{l}512 \times 512 \times 30 \\
(0.390-0.430,0.390-0.430,5) \\
{[\mathrm{TR}=9.6-12 \mathrm{~s}, \mathrm{TE}=121.856-126.784 \mathrm{~ms}]}\end{array}$ & $\begin{array}{l}224-256 \times 256 \times 181(0.82,0.82,2.2-7) \\
{[T R=9 \mathrm{~s}, \mathrm{TE}=86 \mathrm{~ms}]}\end{array}$ & $\begin{array}{l}512 \times 512 \times 27(0.4492,0.4492,5.5) \\
{[\mathrm{TR}=8,800-9,500 \mathrm{~s}, \mathrm{TE}=94.952-96.976 \mathrm{~ms}]}\end{array}$ & $\begin{array}{l}512 \times 512 \times 80(0.469,0.469,2) \\
{[\mathrm{TR}=11 \mathrm{~s}, \mathrm{TE}=125 \mathrm{~ms}]}\end{array}$ \\
\hline T2_FSE & $\begin{array}{l}512 \times 512 \times 28-32 \\
(0.391-0.430,0.391-0.430,5) \\
{[T R=3.504-5.508 \mathrm{~s}, \mathrm{TE}=86.24-98.44 \mathrm{~ms}]}\end{array}$ & & $\begin{array}{l}512 \times 512 \times 21-25 \\
(0.410-0.449,0.410-0.449,6) \\
{[\mathrm{TR}=4.056-6.944 \mathrm{~s}, \mathrm{TE}=81-108.756 \mathrm{~ms}]}\end{array}$ & & $\begin{array}{l}280-292 \times 320 \times 227(0.656,0.656,7) \\
{[\mathrm{TR}=4-4.3 \mathrm{~s}, \mathrm{TE}=95 \mathrm{~ms}]}\end{array}$ & $\begin{array}{l}512 \times 512 \times 27-29 \\
(0.43-0.449,0.43-0.449,5.5) \\
{[T R=4.967-6.997 \mathrm{~s}, \mathrm{TE}=103.404-109.272 \mathrm{~ms}]}\end{array}$ & \\
\hline fMRI & $\begin{array}{l}128 \times 128 \times 30-34 \times 200(1.875,1.875,4.5) \\
{[\mathrm{TR}=2 \mathrm{~s}, \mathrm{TE}=30 \mathrm{~ms}]}\end{array}$ & $\begin{array}{l}128 \times 128 \times 35 \times 100(2.875,2.875,4) \\
{[\mathrm{TR}=3,000 \mathrm{~s}, \mathrm{TE}=35 \mathrm{~ms}]}\end{array}$ & $\begin{array}{l}64 \times 64 \times 6,600-7,600 \\
(3-3.75,3-3.75,3.5-4.5) \\
{[\mathrm{TR}=1.825-2.075 \mathrm{~s}, \mathrm{TE}=29-30 \mathrm{~ms}]}\end{array}$ & $\begin{array}{l}128 \times 128 \times 6,200-7,200(1.875,1.875,4.5) \\
{[\mathrm{TR}=2 \mathrm{~s}, \mathrm{TE}=30 \mathrm{~ms}]}\end{array}$ & & & $\begin{array}{l}128 \times 128 \times 35 \times 100(2.875,2.875,4) \\
{[\mathrm{TR}=3,000 \mathrm{~s}, \mathrm{TE}=35 \mathrm{~ms}]}\end{array}$ \\
\hline DTI & $\begin{array}{l}256 \times 256 \times 67-77 \times 25(0.875,0.875,2) \\
{[\mathrm{TR}=10.771 \mathrm{~s}, \mathrm{TE}=15.541 \mathrm{~ms}]}\end{array}$ & $\begin{array}{l}128 \times 128 \times 70 \times 46(1.719,1.719,2) \\
{[\mathrm{TE}=60 \mathrm{~ms}]}\end{array}$ & $\begin{array}{l}256 \times 256 \times 1,984-2,325 \\
(0.899-1.016,0.899-1.016,2) \\
{[\mathrm{TR}=10-15 \mathrm{~s}, \mathrm{TE}=75.2-88.6 \mathrm{~ms}]}\end{array}$ & $\begin{array}{l}256 \times 256 \times 1,725-2,000(0.875,0.875,2) \\
{[\mathrm{TR}=13.686-15.628 \mathrm{~s}, \mathrm{TE}=74.9-89.3 \mathrm{~ms}]}\end{array}$ & & & $\begin{array}{l}128 \times 128 \times 70 \times 46(1.719,1.719,2) \\
{[\mathrm{TE}=60 \mathrm{~ms}]}\end{array}$ \\
\hline PET & $\mathrm{A}[\mathrm{GE}]$ & $\mathrm{B}[\mathrm{GE}]$ & $\mathrm{C}[\mathrm{GE}]$ & $\mathrm{D}$ [GE] & E [Siemens] & $\mathrm{F}$ [GE] & $\mathrm{G}[\mathrm{GE}]$ \\
\hline
\end{tabular}

$(1.9531-2,1.9531-2)$ $128 \times 128 \times 188(2,2)$

$128 \times 128 \times 47(2.344,2.344)$

$128 \times 128 \times 47(1.9531-2,1.9531-2)$

$128 \times 128 \times 148(1.9531-2,1.9531-2) \quad 256 \times 256 \times 47(0.977,0.977)$

$128 \times 128 \times 188(2,2)$ 
Supplementary Table 2. Demographic characteristics of the study participants according to cognitive status and neuroimaging biomarkers $(\mathrm{N}=640)$

\begin{tabular}{|c|c|c|c|c|c|c|}
\hline $\begin{array}{c}\text { Participant } \\
\text { characteristics }\end{array}$ & $\begin{array}{c}\mathrm{CN} \\
(\mathrm{N}=199)\end{array}$ & $\begin{array}{c}\text { ADCI } \\
(\mathrm{N}=159)\end{array}$ & $\begin{array}{c}\text { VCI } \\
(\mathrm{N}=78)\end{array}$ & $\begin{array}{l}\text { Others } \\
(\mathrm{N}=204)\end{array}$ & $\mathrm{p}^{\dagger}$ & $\mathrm{p}<0.05^{\ddagger}$ \\
\hline Age, years & $71.1 \pm 7.5$ & $73.6 \pm 7.8$ & $74.0 \pm 7.5$ & $72.6 \pm 7.1$ & 0.001 & $\mathrm{a}, \mathrm{b}$ \\
\hline Female & $143(71.9)$ & $97(61.0)$ & $58(74.4)$ & $135(66.2)$ & 0.042 & \\
\hline Education, years & $7.9 \pm 4.7$ & $9.2 \pm 5.0$ & $7.1 \pm 4.9$ & $7.8 \pm 4.7$ & 0.003 & $\mathrm{a}, \mathrm{c}$ \\
\hline Hypertension & $111(55.8)$ & $82(51.6)$ & $48(61.5)$ & $109(53.4)$ & 0.508 & \\
\hline Diabetes & $36(18.1)$ & $31(19.5)$ & $29(37.2)$ & $50(24.5)$ & 0.005 & \\
\hline Dyslipidemia & $88(44.2)$ & $50(31.5)$ & $33(42.3)$ & $76(37.3)$ & 0.082 & \\
\hline APOE e4 carrier & $33(16.6)$ & $86(54.1)$ & $15(19.2)$ & $59(28.9)$ & $<0.001$ & \\
\hline MMSE (0-30) & $26.6 \pm 2.5$ & $19.9 \pm 5.2$ & $20.5 \pm 4.5$ & $23.2 \pm 4.4$ & $<0.001$ & $\mathrm{a}, \mathrm{b}$ \\
\hline S-IADL & $5.6 \pm 5.2$ & $15.4 \pm 10.8$ & $12.9 \pm 10.3$ & $9.9 \pm 8.2$ & $<0.001$ & $\mathrm{a}, \mathrm{b}$ \\
\hline SGDS-K & $6.1 \pm 4.8$ & $5.4 \pm 4.8$ & $7.7 \pm 5.5$ & $7.0 \pm 5.2$ & 0.005 & $b, c$ \\
\hline GDS & $2.7 \pm 0.6$ & $4.1 \pm 1.0$ & $3.7 \pm 0.9$ & $3.3 \pm 1.0$ & $<0.001$ & \\
\hline $\mathrm{A} \beta$ deposition on PET & $8(4.0)$ & $136(85.5)$ & $5(6.4)$ & $71(34.8)$ & $<0.001$ & \\
\hline Schelten's scale & $1.3 \pm 0.8$ & $2.1 \pm 0.8$ & $2.1 \pm 0.8$ & $1.8 \pm 0.8$ & $<0.001$ & $\mathrm{a}, \mathrm{b}$ \\
\hline WMH on MRI & & & & & $<0.001$ & \\
\hline Minimal & $138(69.4)$ & $94(59.1)$ & $0(0.0)$ & $148(72.6)$ & & \\
\hline Moderate & $61(30.7)$ & $65(40.9)$ & $59(75.6)$ & $24(11.8)$ & & \\
\hline Severe & $0(0.0)$ & $0(0.0)$ & $19(24.4)$ & $32(15.7)$ & & \\
\hline
\end{tabular}

Data are shown as the mean \pm SD or number (\%). ${ }^{\dagger}$ analysis of variance or chi-square test; ${ }^{\ddagger}$ tukey post hoc analysis. a, NC vs. ADCI; b, NC vs. VCI; c, ADCI vs. VCI. CN, cognitively normal; ADCI, AD-related cognitive impairment; VCI, vascular cognitive impairment; NOS, not otherwise specified due to mixed pathology; APOE, apolipoprotein E; MMSE, mini-mental state examination; S-IADL, Seoul instrumental activities of daily living; SGDS-K, Korean version of the short form of geriatric depression scale; GDS: global deterioration scale; WMH: white matter hyperintensities 
Supplementary Table 3. Neuropsychological test results of the study participants according to cognitive status and neuroimaging biomarkers $(N=640)$

\begin{tabular}{|c|c|c|c|c|c|c|}
\hline Neuropsychological test & $\mathrm{CN}(\mathrm{N}=199)$ & $\operatorname{ADCI}(\mathrm{N}=159)$ & $\mathrm{VCI}(\mathrm{N}=78)$ & $\operatorname{NOS}(\mathrm{N}=204)$ & $\mathrm{p}^{\dagger}$ & $\mathrm{p}<0.05^{\ddagger}$ \\
\hline \multicolumn{7}{|l|}{ Attention function } \\
\hline Digit span test-backward & $-0.13(1.01)$ & $-0.99(1.34)$ & $-0.69(0.98)$ & $-0.49(1.07)$ & $<0.001$ & $\mathrm{a}, \mathrm{b}$ \\
\hline \multicolumn{7}{|l|}{ Language function } \\
\hline Boston maming test & $-0.04(1.07)$ & $-1.32(1.67)$ & $-1.09(1.66)$ & $-0.83(1.72)$ & $<0.001$ & $a, b$ \\
\hline \multicolumn{7}{|l|}{ Visuospatioal function } \\
\hline RCFT-copy & $-0.30(1.13)$ & $-2.33(2.85)$ & $-0.75(5.56)$ & $-1.02(1.79)$ & $<0.001$ & $\mathrm{a}, \mathrm{c}$ \\
\hline \multicolumn{7}{|l|}{ Memory function } \\
\hline SVLT-delayed recall & $-0.35(1.09)$ & $-1.98(0.93)$ & $-1.13(1.12)$ & $-1.04(1.16)$ & $<0.001$ & $a, b, c$ \\
\hline RCFT-delayed recall & $-0.18(0.98)$ & $-1.62(0.88)$ & $-0.95(0.90)$ & $-0.61(1.08)$ & $<0.001$ & $a, b, c$ \\
\hline \multicolumn{7}{|l|}{ Frontal/executive function } \\
\hline Stroop test-color reading & $-0.22(1.00)$ & $-1.80(1.63)$ & $-1.48(1.49)$ & $-1.03(1.51)$ & $<0.001$ & $\mathrm{a}, \mathrm{b}$ \\
\hline COWAT-phonemic task & $-0.44(0.89)$ & $-1.19(1.10)$ & $-1.18(0.84)$ & $-0.68(0.98)$ & $<0.001$ & $a, b$ \\
\hline
\end{tabular}

${ }^{\dagger}$ analysis of variance; ${ }^{\dagger}$ tukey post hoc analysis. a, NC vs. ADCI; b, NC vs. VCI; c, ADCI vs. VCI. CN, cognitively normal; ADCI, AD-related cognitive impairment; VCI, vascular cognitive impairment; NOS, not otherwise specified due to mixed pathology; RCFT, Rey complex figure test; SVLT, Seoul verbal learning test; COWAT, controlled oral word association test-phonemic task 